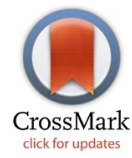

www.renhyd.org

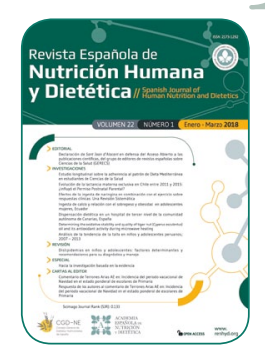

EDITORIAL

\title{
Declaración de Sant Joan d'Alacant en defensa del Acceso Abierto a las publicaciones científicas, del grupo de editores de revistas españolas sobre Ciencias de la Salud (GERECS)
}

\author{
Grupo de Editores de Revistas Españolas sobre Ciencias de la Salud (GERECS)
}

Editor Asignado: Rodrigo Martínez-Rodríguez. Universidad de Murcia. Murcia, España.

Recibido el 25 de noviembre de 2017; aceptado el 15 de marzo de 2018; publicado el 18 de marzo de 2018.

Este documento se publica simultáneamente en las revistas que suscribieron la Declaración de Sant Joan d'Alacant del Grupo de Editores de Revistas Españolas sobre Ciencias de la Salud (GERECS) el día 25 de noviembre de 2017.

\section{CITA}

Grupo de Editores de Revistas Españolas sobre Ciencias de la Salud (GERECS). Declaración de Sant Joan d'Alacant en defensa del Acceso Abierto a las publicaciones científicas, del grupo de editores de revistas españolas sobre Ciencias de la Salud (GERECS). Rev Esp Nutr Hum Diet. 2018; 22(1): 1-3. doi: 10.14306/renhyd.22.1.569

El concepto de Acceso Abierto (Open Access, OA) no sólo tiene que ver con la accesibilidad al documento científico, sino también con los permisos de reutilización más o menos restrictivos en función de los derechos reservados para su distribución. A partir de esta idea, surgieron numerosas iniciativas, con o sin ánimo de lucro, con el fin de facilitar el acceso universal a través de internet a las publicaciones científicas.

Proyectos como Scientific Electronic Library Online (SciELO, 1998), The Scholarly Publishing and Academic Resources Coalition (SPARC, 1998), PubMed Central (PMC, 2000), The Public Library of Science (PLOS, 2000) o BioMed Central (BMC, 2001), fueron pioneros de una revolución que haría replantear las estrategias comerciales de la edición científica. Otros como Dialnet (2001), Red de Revistas Científicas de América Latina y el Caribe, España y Portugal (Redalyc, 2003) y el Directory of Open Access Journals (DOAJ, 2003), también extenderían el movimiento del acceso abierto y ayudaron al proceso de globalización del conocimiento en las comunidades científicas del ámbito iberoamericano.
Las primeras Declaraciones que sentaron las bases del futuro desarrollo del acceso abierto fueron: la Budapest Open Access Initiative (2002), Berlin Declaration on Open Access to Knowledge in the Sciences and Humanities (2003) y Bethesda Statement on Open Access Publishing (2003), esta última considerada como la declaración de principios para las Ciencias de la Salud.

Por otro lado, se han promovido manifiestos, impulsados generalmente en reuniones de editores de revistas científicas que proponían algunas recomendaciones para el correcto desarrollo del acceso abierto a la ciencia. En España se podría citar la Declaración de la Alhambra (2010), que aportó recomendaciones para las políticas y plan de acción para el desarrollo del acceso abierto en el sur de Europa. Más reciente y en el ámbito latinoamericano, se elaboró la Declaración de la reunión de Consorcios de Iberoamérica y el Caribe (2017), que entre sus recomendaciones discute la desviación del concepto de Open Access por la creciente aparición de revistas de pago por publicación con precios a 
Declaración de Sant Joan d'Alacant en defensa del Acceso Abierto a las publicaciones científicas, del grupo de editores de revistas españolas sobre Ciencias de la Salud (GERECS)

veces abusivos (Article Processing Charges, APC, tasas de procesamiento de artículos) con la etiqueta de Acceso Abierto.

La pasada conferencia de Ámsterdam, "Open Science - From Vision to Action" (2016) formuló dos importantes objetivos paneuropeos a alcanzar en el año 2020:

- Acceso Abierto completo para todas las publicaciones científicas.

- Un nuevo enfoque orientado hacia la reutilización óptima de los datos de investigación.

Para alcanzar estos objetivos, se propuso la aplicación de nuevos sistemas de evaluación y recompensa de los trabajos científicos y la generación de políticas de buenas prácticas.

En esta línea, los ministros de ciencia de las naciones de la Unión Europea acordaron, en la sesión celebrada el 27 de mayo de 2016, el documento The transition towards an Open Science system - Council conclusions, recomendando que las publicaciones resultantes de la investigación financiadas con fondos públicos estén disponibles de forma gratuita en el año 2020, para lo cual, cada país deberá implementar su propia política de publicación.

Este acuerdo subraya que el principio para la reutilización óptima de los datos de investigación debería ser "lo más abierto posible, tan cerrado como sea necesario" y hace hincapié en que las oportunidades para la reutilización óptima de los datos de investigación sólo pueden realizarse si los datos son consistentes con los principios FAIR (Findable, Accessible, Interoperable and Re-usable - encontrables, accesibles, interoperables y reutilizables) dentro de un entorno seguro y confiable.

Así, la European Open Science Policy Platform, en su tercera reunión de marzo de 2017, adoptaba las siguientes recomendaciones:

- Las comunidades interesadas, los Estados miembros y la Comisión Europea deberían evaluar e identificar conjuntamente cómo se debe alcanzar el mandato de Acceso Abierto para 2020.

- El progreso hacia un AA completo debe tener en cuenta la rapidez con la que cambia el sistema de publicación y cómo las comunicaciones académicas crecen en riqueza y variedad.

- No hay una solución única, aunque el objetivo final para todas las disciplinas pueda ser el mismo. Las cuestiones relacionadas con el cumplimiento, incluidos los incentivos y la observancia, deberían proponerse, aclararse y armonizarse de una manera que sea sensible a todas las disciplinas.

- Las opciones de las condiciones de pago por la publicación deben ser claras y de fácil localización en las condiciones establecidas por cada revista.

- A partir de 2020 la Comisión Europea debe avanzar hacia una definición más amplia de $A A$, que incorpore toda la gama de formatos y aplicaciones emergentes como resultado de la investigación científica.

Teniendo en cuenta todo lo anteriormente mencionado, conscientes de los futuros cambios que tendrán que asumir los editores de las revistas españolas sobre Ciencias de la Salud, éstos proponen las siguientes recomendaciones y peticiones:

1. Adherirse a los criterios emanados de la reunión de marzo de 2017 de la European Open Science Policy Platform.

2. Alentar a nuestras instituciones a que respalden la Expresión de Interés OA2020 (https://oa2020.org/) y, en consecuencia, firmen sus principios.

3. Instar a las agencias de investigación a nivel nacional a poner en marcha políticas científicas que requieran a sus investigadores que depositen sus publicaciones en repositorios institucionales.

4. Teniendo en cuenta el compromiso social de las revistas en AA con la accesibilidad del conocimiento, incluyendo a la ciudadanía, se solicita el reconocimiento como mérito académico/profesional la publicación en revistas de acceso abierto que estén indizadas en plataformas comprometidas con la excelencia, como SciELO, Redalyc o DOAJ.

Asimismo, en línea con la Declaración de San Francisco de Evaluación de la Investigación (San Francisco Declaration on Research Assesment, DORA, 2012), los editores de revistas de Ciencias de la Salud consideran necesario apoyar la adopción de las siguientes prácticas:

1. Reducir el énfasis del índice de impacto, u otras métricas basadas en indicadores sobre la revista en que fue publicado, como una herramienta de promoción personal.

2. Promover nuevos indicadores relacionados con el contenido científico del artículo en lugar de métricas sobre la revista en que fue publicado.

\section{Sant Joan d'Alacant, España, 25 de noviembre de 2017.}

\section{Firmantes:}

- Javier Sanz-Valero

Editor jefe de la revista Medicina y Seguridad del Trabajo. Instituto de Salud Carlos III, Escuela Nacional de Medicina del Trabajo, Madrid, España.

- Remedios Melero Melero Miembro del grupo de trabajo Acceso Abierto a La Ciencia. Instituto de Agroquímica y Tecnología de Alimentos-CSIC, Valencia, España.

- Cristina Bojo Canales

Coordinadora de la red SciELO España. Instituto de Salud CarIos III, Biblioteca Nacional de Ciencias de la Salud, Madrid, España. 
Declaración de Sant Joan d'Alacant en defensa del Acceso Abierto a las publicaciones científicas, del grupo de editores de revistas españolas sobre Ciencias de la Salud (GERECS)

- Enrique Perdiguero Gil

Director de la revista Doctorado. Universidad Miguel Hernández, Elche, España.

- Jesús Manuel Culebras Fernández

Director de la revista Journal of Negative and No Positive Results. Asociación para el Progreso de la Medicina, León, España.

- Carmina Wanden-Berghe

Directora de la revista Hospital a Domicilio. Centro Internacional Virtual de Investigación en Nutrición (CIVIN), Alicante, España.

- Maria Dolores Ruiz López

Editora de la revista Ars Pharmaceutica. Universidad de Granada, Granada, España.

- Manuel Amezcua Martínez

Director de la revista Index de Enfermería. Fundación Index, Granada, España.

- Carlos Alvarez-Dardet

Director de la revista Gaceta Sanitaria. Sociedad Española de Salud Pública (SESPAS), Barcelona, España.

- Mikel Astrain Gallart

Director de la revista Dynamis. Universidad de Granada, Granada, España.

- María del Mar Vaquero Pérez

Directora de la revista Cirugía Plástica Ibero-Latinoamericana. Federación Ibero-latinoamericana de Cirugía Plástica (FILACP) y Sociedad Española de Cirugía Plástica, Reparadora y Estética (SECPRE), Madrid, España.

- María José López Montesinos

Directora de la revista Enfermería Global. Universidad de Murcia, Murcia, España.

- Manuel Sosa Henríquez

Director de la revista Osteoporosis y Metabolismo Mineral. Sociedad Española de Investigación Ósea y Metabolismo Mineral (SEIOMM), Madrid, España.

- José Luis Pardal-Refoyo Director de la revista ORL. Universidad de Salamanca, Salamanca, España.

- Agustín Romero Medina

Director de la revista Anales de Psicología. Universidad de Murcia, Murcia, España.

- Elena Primo Peña

Directora de la Biblioteca Nacional de Ciencias de la Salud. Instituto de Salud Carlos III, Madrid, España.

- Ángel Hernández Merino

Director de la Revista Pediatría de Atención Primaria. Asociación Española de Pediatría de Atención Primaria, Madrid, España.
- Luis Miguel Torres Morera

Director de la Revista de la Sociedad Española del Dolor. Sociedad Española del Dolor, Cádiz, España.

- Antonio Bascones Martínez

Director de la revista Avances en Odontoestomatología. Ediciones Avances, Madrid, España.

- Fernando Fernandez-Llimos

Editor jefe de la revista Pharmacy Practice. Centro de Investigaciones y Publicaciones Farmacéuticas, Granada, España.

- Mariano Rodriguez Portillo

Director de la revista Nefrología. Sociedad Española de Nefrología, Santander, España.

- Javier Soldevilla Agreda

Director de la revista GEROKOMOS. Sociedad Española de Enfermería Geriátrica y Gerontológica, Barcelona, España.

- José Miguel Soriano del Castillo

Editor jefe de la Revista Española de Nutrición Humana y Dietética. Fundación Academia Española de Nutrición y Dietética, Pamplona, España.

- Elena Ronda Pérez

Directora de la revista Archivos de Prevención de Riesgos Laborales. Societat Catalana de Salut Laboral, Barcelona, España.

- Martín Rodríguez Álvaro

Director de ENE Revista de Enfermería. Grupo de Enfermería ENE, La Palma, España.

- Jose Manuel Moreno Villares

Director de la revista Nutrición Hospitalaria. Sociedad Española de Nutrición Parenteral y Enteral (SENPE), Madrid, España.

- Teresa del Campo Balsa

Directora de la Revista de la Asociación Española de Especialistas en Medicina del Trabajo. Asociación Española de Especialistas en Medicina del Trabajo, Madrid, España.

- Marta Molina Olivas

Editora de la Revista Española de Salud Pública. Ministerio de Sanidad, Servicios Sociales e Igualdad. Madrid, España.

- Julián Almaraz Carretero

Director de la revista Escritos de Psicología. Facultad de Psicología de la Universidad de Málaga, Málaga, España.

- Ernest Abadal

Editor de la revista BiD. Universitat de Barcelona, Barcelona, España.

- Rodolfo Crespo Montero

Director de la revista Enfermería Nefrológica. Sociedad Española de Enfermería Nefrológica, Madrid, España.

- Ana Dago Martínez

Directora de la revista Pharmaceutical Care España. Fundación Pharmaceutical Care España, Madrid, España. 